\title{
The synchronous generator based on a hybrid excitation with the extended range of voltage adjustment
}

\author{
Andrzej Popenda ${ }^{1, *}$, and Stawomir Chwalba ${ }^{1}$ \\ ${ }^{1}$ Czestochowa University of Technology, Department of Electrical Engineering, Al. Armii Krajowej 17, \\ 42-200 Czestochowa, Poland
}

\begin{abstract}
In the paper a synchronous generator based on a hybrid excitation, including permanent magnets and field coil, is studied. The structures of rotor magnetic circuit allowing one to expand the range of generator voltage adjustment are explained and compared with the standard hybrid rotor. The structures are described mathematically and their equivalent circuits are given. The results of theoretical analysis and experimental test are presented.
\end{abstract}

\section{Introduction}

Increasing the efficiency of electrical machines is a global trend aimed at the environmental protection. As a result of these efforts the conventional electrical machines, among which are induction machines and synchronous machines excited by field coil, are being replaced by permanent magnet machines (PMM) [1-5]. Recently, the interest in these machines has increased substantially due to the decrease in their prices. The use of PMM in appliances demanding a low rotational speed allows one to eliminate the gearbox, which connects the working machinery and the standard induction motor or the slow-moving turbine to the standard synchronous generator. The use of gearbox is costly, decreases efficiency of device as well requires maintenance. Low speed of PMM means a lot of magnetic poles and, as a consequence, results in a significant diameter of these machines. Currently, the PMM are most frequently being used as ship drives and wind power generators.

An important requirement expected from a synchronous generator, that supplies power to an individual load, is to maintain a constant frequency and a constant voltage at the output terminals of armature winding despite the load variations. In the conventional generators excited by field coil such working conditions are achieved by using speed and voltage controllers. The operation of voltage controller is defined in terms of regulation characteristics, which show how the exciting current has to be set in order to maintain the constant armature voltage at constant power factor an constant rotational speed despite the load current variations. Permanent magnet synchronous generators (PMSG) do not have the possibility of exciting field regulation and their voltage variation exceeds twenty percent.

*Corresponding author: popenda@el.pcz.czest.pl 


\section{The concepts of synchronous generator based on a hybrid excitation}

One of the methods to reduce the range of PMSG voltage variations, caused by load current changes, is the use of self-stabilization as a consequence of improvement of generator external characteristics. Such conditions can be achieved for example by designing the appropriate construction of rotor, in which the surface mounted permanent magnets (PM), relatively simple in practical implementation, are replaced by interior mounted permanent magnets, which allow to decrease the voltage variation to around $10 \%$.

More effective stabilization of generator voltage may be ensured by the use of additional field coil. In this solution the main excitation is based on permanent magnets, which allow to maintain the high efficiency of generator. The aim of additional field coil is the adjustment of generator voltage in a certain range, which allows one to compensate the voltage drop caused by the increase of load current, at the cost of a modest reduction of generator efficiency. There are some ways of implementation of synchronous generator with a voltage stability. One of the solution is the rotor with the hybrid parallel magnetic circuit based on two independent parts assembled on a common shaft (Fig. 1). The following relationship between the lengths of the respective rotor parts: $l_{\mathrm{m}}=3 l_{\mathrm{e}}$ was assumed in the paper [2].

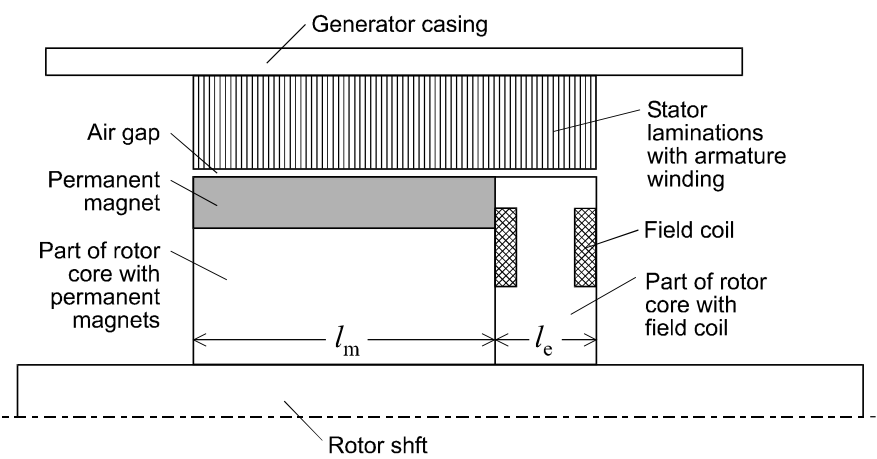

Fig. 1. Explanatory figure of synchronous generator based on the hybrid parallel excitation including permanent magnets and field coil, where $l_{\mathrm{m}}=3 l_{\mathrm{e}}[2]$.

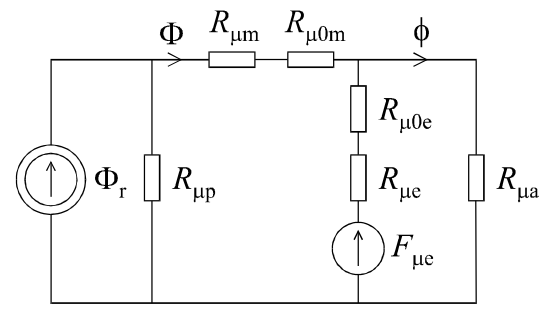

Fig. 2. Equivalent circuit of magnetic core of no-load synchronous generator based on the hybrid parallel excitation (Fig. 1), where $\Phi_{\mathrm{r}}$ is the magnetic flux corresponding to the PM remanence, $\Phi$ is the magnetic flux in part of rotor core with permanent magnets, $\phi$ is the rotor magnetic flux permeating stator laminations, $F_{\mu \mathrm{e}}$ is the equivalent magnetomotive force of field coil, $R_{\mu \mathrm{p}}$ is the equivalent reluctance of permanent magnet and path of PM leakage magnetic flux, $R_{\mu \mathrm{m}}$ is the equivalent reluctance of rotor core part with permanent magnets and path of its leakage magnetic flux, $R_{\mu 0 \mathrm{~m}}$ is the reluctance of air gap at rotor core part with permanent magnets, $R_{\mu \mathrm{e}}$ is the equivalent reluctance of rotor core part with field coil and path of its leakage magnetic flux, $R_{\mu 0 \mathrm{e}}$ is the reluctance of air gap at rotor core part with field coil, $R_{\mu \mathrm{a}}$ is the equivalent reluctance of stator laminations including yoke and teeth and path of stator leakage magnetic flux. 
The equivalent circuit of magnetic core of no-load synchronous generator based on the hybrid parallel excitation (Fig. 1) is shown in Fig. 2.

The explanatory figure of modified synchronous generator based on the extended hybrid excitation is shown in Fig. 3. In the proposed solution the common part, being added to the rotor core, is penetrated by both magnetic fluxes i.e. excited by permanent magnets and field coil, respectively.

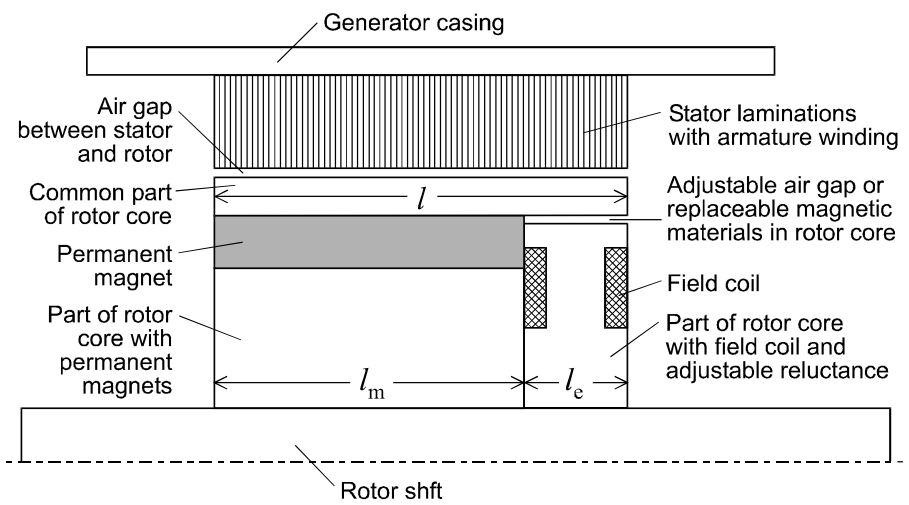

Fig. 3. Explanatory figure of modified synchronous generator based on the extended hybrid excitation.

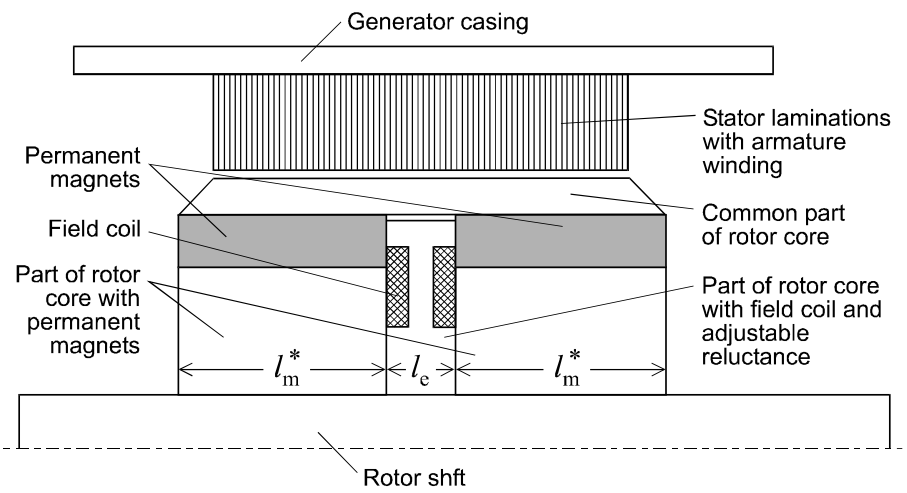

Fig. 4. Explanatory figure of the other variant of modified synchronous generator based on the extended hybrid excitation $(*)$.

The other variant of the modified synchronous generator based on the extended hybrid excitation is shown in Fig. 4. As a consequence of increasing the working area of permanent magnets by $50 \%\left(l_{\mathrm{m}}{ }^{*}=0.75 l_{\mathrm{m}}\right)$ the magnetic flux, permeating the part of rotor magnetic core with permanent magnets, was increased to $\Phi^{*}=1.5 \Phi$. In the proposed solution the flux is higher as a result of increasing the axial length of rotor core without increasing the length of stator stack of laminations. The use of permanent magnets with the higher remanence may lead to the similar effect without increasing the length of rotor core. The area of part of rotor core with field coil may also be decreased due to the relatively high reluctance of this part of core. As a consequence, the length of winding wire and its resistance as well as the power loss in excitation winding may be lower.

The equivalent circuit of magnetic core of no-load modified synchronous generators based on the extended hybrid excitation (Figs. 3 and 4) is shown in Fig. 5. 


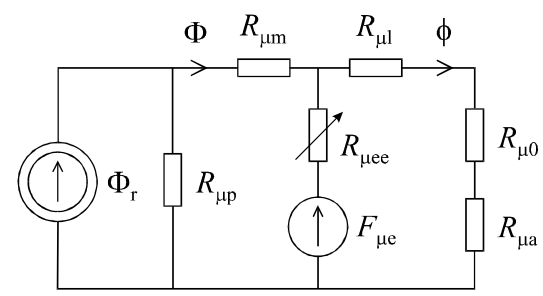

Fig. 5. Equivalent circuit of magnetic core of no-load modified synchronous generators based on the extended hybrid excitation (Figs. 3 and 4), where $R_{\text {uee }}$ is the adjustable reluctance of rotor core part with field coil, $R_{\mu l}$ is the reluctance of common part of rotor core, $R_{\mu 0}$ is the reluctance of entire air gap between stator and rotor.

Adjustment of air gap or replacement of magnetic materials in part of rotor core with field coil (Figs. 3 and 4) result in a change of reluctance $R_{\mu \text { ee }}$ (Fig. 5).

\section{The analysis of synchronous generators based on a hybrid excitation}

The synchronous generators based on a hybrid excitation presented in the previous chapter have been analyzed. The analysis assumed that magnetic circuits of synchronous generators are unsaturated. It is known that $R_{\mu 0}>>R_{\mu \mathrm{e}}$ and $R_{\mu 0}>>R_{\mu \mathrm{m}}$ and $R_{\mu 0}>>R_{\mu \mathrm{a}}$ and $R_{\mu 0}>>R_{\mu \mathrm{l}}$ as a consequence of significant differences in magnetic permeability of ferromagnetic materials and air. Thus, the respective reluctances of ferromagnetic parts may be omitted from the considerations: $R_{\mu \mathrm{e}} \approx 0, R_{\mu \mathrm{m}} \approx 0, R_{\mu \mathrm{a}} \approx 0, R_{\mu \mathrm{l}} \approx 0$. The abovementioned assumption lead to the following dependencies between rotor magnetic flux permeating stator laminations $\phi$ and magnetic flux in part of rotor core with permanent magnets $\Phi$ as well as magnetomotive force (MMF) of field coil $F_{\mu \mathrm{e}}$ :

- for the equivalent circuit of synchronous generator based on the hybrid parallel excitation (Fig. 2)

$$
\phi=\Phi+F_{\mu \mathrm{e}} / R_{\mu 0 \mathrm{e}}
$$

- for the equivalent circuit of modified synchronous generators based on the extended hybrid excitation (Fig. 5)

$$
\phi=\left(R_{\mu \mathrm{ee}} \Phi+F_{\mu \mathrm{e}}\right)\left(R_{\mu \mathrm{ee}}+R_{\mu 0}\right)^{-1}
$$

where: $R_{\mu 0 \mathrm{e}}=R_{\mu 0}\left(l_{\mathrm{e}}+l_{\mathrm{m}}\right) / l_{\mathrm{e}}=4 R_{\mu 0}$.

The abovegiven dependencies can be simpler and more enlightening if the relative quantities are introduced: $\phi^{\mathrm{r}}=\phi / \Phi, \Phi^{\mathrm{r}}=1, \Phi^{* \mathrm{r}}=1.5, F_{\mu \mathrm{e}}{ }^{\mathrm{r}}=F_{\mu \mathrm{e}} / R_{\mu 0} \Phi, R_{\mu 0 \mathrm{e}}{ }^{\mathrm{r}}=R_{\mu 0 \mathrm{e}} / R_{\mu 0}$, $R_{\mu \mathrm{ee}}^{\mathrm{r}}=R_{\mu \mathrm{ee}} / R_{\mu 0}$. The dependencies between relative rotor magnetic flux permeating stator laminations $\phi^{\mathrm{r}}$ and relative magnetic flux in part of rotor core with permanent magnets $\Phi^{\mathrm{r}}$ or $\Phi^{{ }^{* \mathrm{r}}}$ as well as relative MMF of field coil $F_{\mu \mathrm{e}}{ }^{\mathrm{r}}$ are as follows:

- for the synchronous generator based on the hybrid parallel excitation (Fig. 1)

$$
\phi^{\mathrm{r}}=1+F_{\mu \mathrm{e}}^{\mathrm{r}} / R_{\mu 0 \mathrm{e}}^{\mathrm{r}}=1+F_{\mu \mathrm{e}}^{\mathrm{r}} / 4
$$

- for the modified synchronous generator based on the extended hybrid excitation (Fig. 3)

$$
\phi^{\mathrm{r}}=\left(R_{\mu \mathrm{ee}}^{\mathrm{r}}+F_{\mu \mathrm{e}}^{\mathrm{r}}\right)\left(R_{\mu \mathrm{ee}}^{\mathrm{r}}+1\right)^{-1}
$$


- for the other variant of modified synchronous generator based on the extended hybrid excitation (Fig. 4)

$$
\phi^{\mathrm{r}}=\left(1.5 R_{\mu \mathrm{ee}}^{\mathrm{r}}+F_{\mu \mathrm{e}}^{\mathrm{r}}\right)\left(R_{\mu \mathrm{ee}}^{\mathrm{r}}+1\right)^{-1}
$$

The electromotive force (EMF) induced in armature winding is dependent on magnetic flux permeating stator stack of laminations:

$$
e=N d \phi / d t
$$

where $N$ is number of turns in armature winding. For sinusoidal magnetic flux the rms value of EMF induced in armature winding is as follows:

$$
E=4.44 N f \phi
$$

where $f$ is the frequency of induced voltage. The dependency between relative induced EMF and relative magnetic flux:

$$
E^{\mathrm{r}}=\phi^{\mathrm{r}}
$$

On the basis of the derived dependencies 3, 4, 5 and 8 the graphs of relative EMF induced in armature winding $E^{\mathrm{r}}$ (or relative flux $\phi^{\mathrm{r}}$ ) vs. relative MMF of field coil $F_{\mu \mathrm{e}}{ }^{\mathrm{r}}$ have been constructed for various relative values of adjustable reluctance $R_{\mu \mathrm{ee}}{ }^{\mathrm{r}}$ of rotor core part with field coil (Fig. 6).

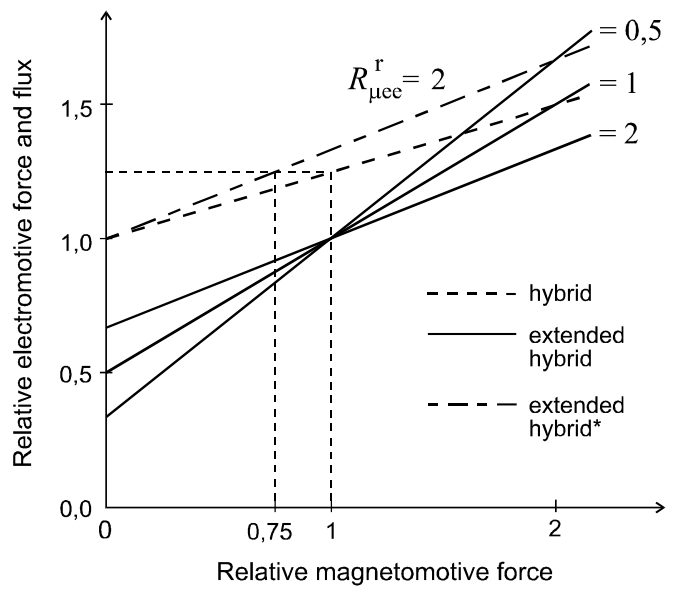

Fig. 6. Graphs of relative EMF induced in armature winding $E^{\mathrm{r}}$ (or relative flux $\phi^{\mathrm{r}}$ ) vs. relative MMF of field coil for various synchronous generators based on a hybrid excitation.

As apparent from the constructed graphs the modification of rotor magnetic core (Figs. 3 and 4) results in the extension of voltage adjustment range: (a) $33 \%$ for $R_{\mu \mathrm{ee}}^{\mathrm{r}}=2$, (b) $50 \%$ for $R_{\mu \mathrm{ee}}{ }^{\mathrm{r}}=1$ and (c) $67 \%$ for $R_{\mu \mathrm{ee}}^{\mathrm{r}}=0.5$, whereas for synchronous generator based on the hybrid parallel excitation (Fig. 1) the voltage adjustment range is equal to $25 \%$. The solution depicted in Fig. 4 allows for the induced voltage $(E)$ for $F_{\mu \mathrm{e}}{ }^{\mathrm{r}}=0$ to be the same as in the case of synchronous generator based on the hybrid parallel excitation (Fig. 1). However, a wider range of voltage adjustment in the case of solution depicted in Fig. 4 allows for the induced voltage for $F_{\mu \mathrm{e}}{ }^{\mathrm{r}}=0.75$ to be the same as in the case of synchronous generator based on the hybrid parallel excitation for $F_{\mu \mathrm{e}}{ }^{\mathrm{r}}=1$ (see Fig. 6). This reduces power loss in field coil of modified synchronous generator depicted in Fig. 4 nearly by half in relation to the synchronous generator based on the hybrid parallel excitation (Fig. 1). 


\section{Results of experimental test}

The physical model of synchronous generator based on the extended hybrid excitation (Fig. 7) has been made in order to carry out experimental investigations. The model generator was based on structural components of induction motor stator i.e. stack of laminations, casing and rotor bearings as well as structural components of claw pole rotor (Fig. 8) adopted from a car alternator. The availability on the market as well as the relatively low price of these components, in contrast to the price of special design components, determined the choice of the abovementioned solution. The constructed model generator corresponds to the equivalent circuit shown in Fig. 5.

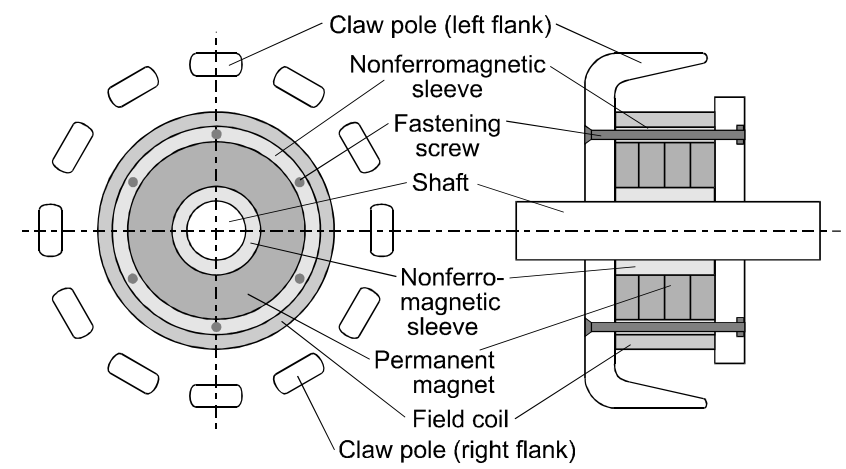

Fig. 7. Explanatory figure of physical model of synchronous generator based on the extended hybrid excitation (axial and cross sections).

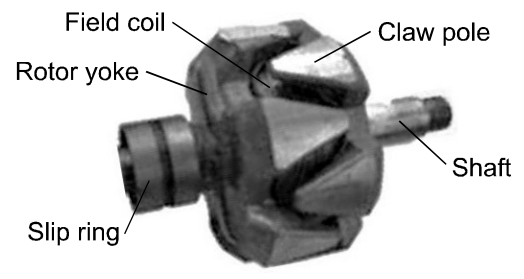

Fig. 8. Claw pole rotor.

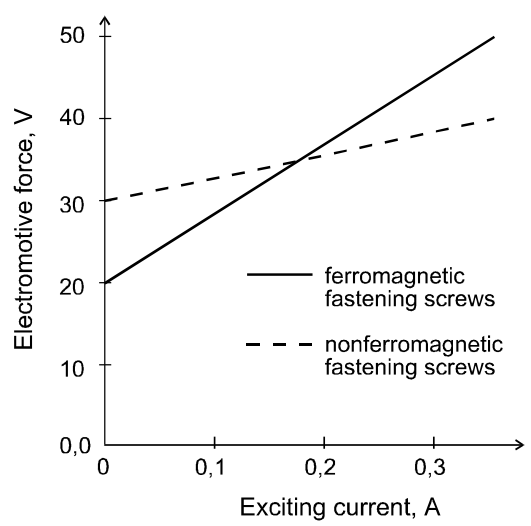

Fig. 9. Graphs of EMF induced in armature winding $E$ vs. exciting current of field coil for the model synchronous generator based on the extended hybrid excitation. 
The fastening screws with different magnetic permeability were used to adjust the reluctance $R_{\text {uee }}$ of rotor core part with field coil. The graphs of induced voltage versus exciting current of field coil have been made for the following cases: (a) ferromagnetic fastening screws and (b) nonferromagnetic fastening screws. The results of experimental test confirm the results of carried out analysis.

\section{Conclusions}

An important requirement expected from a synchronous generator, that supplies power to an individual load, is to maintain a constant frequency and a constant voltage at the output terminals of armature winding despite the load variations. In the conventional generators excited by field coil such working conditions are achieved by using speed and voltage controllers. Permanent magnet synchronous generators do not have the possibility of exciting field regulation and their voltage variation exceeds twenty percent. The effective stabilization of generator voltage may be ensured by the use of additional field coil. In this paper the design solutions of synchronous generator with the modified rotor magnetic core, that allows to extend the range of generator voltage adjustment while reducing the power loss in the exciting winding of field coil, are proposed. The results of carried out analysis and experimental test confirmed the positive properties of the proposed solutions.

\section{References}

1. A. Popenda, S. Chwalba, Przegl. El., 12, 265-268 (2014)

2. S.A. Gawron, Przegl. El., 12, 299-304 (2014)

3. J. Kolehmainen, J. Ikäheimo, IEEE Trans. on Energy Conv., 23, 86-91 (2008)

4. Y. Amara, L. Vido, M. Gabsi, E. Hoang, A. Hamid Ben Ahmed, M. Lecrivain, IEEE Trans. on Veh. Tech., 58, 2137-2149 (2009)

5. H. Lin, X. Liu, Z.Q. Zhu, S. Fang, IET El. Power App., 5, 628-635 (2011) 